\title{
Human-Autonomy Teaming and Agent Transparency
}

Jessie Y.C. Chen, PhD

US Army Research Laboratory

Orlando, FL 32826, USA

jessie.chen@us.army.mil

Michael J. Barnes

Ft. Huachuca, AZ, USA

michael.j.barnes.civ@mail.mil
US Army Research Laboratory

\author{
Anthony Selkowitz, PhD \\ Kimberly Stowers \\ Shan Lakhmani \\ Nicholas Kasdaglis \\ Institute for Simulation \\ \& Training \\ University of Central Florida \\ Orlando, FL, USA \\ aselkowi@ist.ucf.edu \\ kstowers@ist.ucf.edu \\ slakhman@ist.ucf.edu \\ nkasdagl@ist.ucf.edu
}

\section{Abstract}

We developed the user interfaces for two Human-Robot Interaction (HRI) tasking environments: dismounted infantry interacting with a ground robot (Autonomous Squad Member) and human interaction with an intelligent agent to manage a team of heterogeneous robotic vehicles (IMPACT). These user interfaces were developed based on the Situation awareness-based

Permission to make digital or hard copies of part or all of this work for personal or classroom use is granted without fee provided that copies are not made or distributed for profit or commercial advantage and that copies bear this notice and the full citation on the first page. Copyrights for thirdparty components of this work must be honored. For all other uses, contac the Owner/Author. Copyright is held by the owner/author(s) UIT Companion, March 07-10, 20

DOl: http //dx doi.org/10.1145/2876456.2879479
Agent Transparency (SAT) model. User testing showed that as agent transparency increased, so did overal human-agent team performance. Participants were able to calibrate their trust in the agent more appropriately as agent transparency increased.

\section{Author Keywords}

Agent transparency; human-agent teaming; humanrobot interaction; situation awareness; autonomy

\section{ACM Classification Keywords}

H.1.2 [User Machine Systems]: Human Factors, Human Information Processing; H.5.2 [User Interfaces]:

Theory \& Methods

\section{Introduction}

We developed a model of agent transparency [4] to support operator situation awareness (SA) of the mission environment involving the agent-the SA-based Agent Transparency (SAT) model-based on the theory of SA [5], the Beliefs-Desires-Intentions Agent Framework [9], and other relevant previous work [3][7] (Fig. 1). Agent transparency is defined as the "quality of an interface pertaining to its abilities to afford an operator's comprehension about an intelligent agent's intent, performance, future plans, and reasoning process" [4]. At the first level of the SAT model, the operator is provided with the basic 


\section{Situation awareness- based Agent Transparency (SAT) \\ Model}

Level 1: Agent's current status/actions/plans

- Purpose: Desire (Goal selection)

- Process: Intentions (Planning/Execution);

Progress

- Performance

Level 2: Agent's reasoning process

- Reasoning process

(Belief/Purpose)

- Environmental \& other constraints/affordances

\section{Level 3: Agent's}

projections/predictions

- Projection of future

outcomes

- Uncertainty and potential

limitations; Likelihood of

success/failure

- History of Performance

Figure 1: SAT Model [1] information about the agent's current state and goals, intentions, and proposed actions. At the second level, the operator is provided information about the agent's reasoning process behind those actions and the constraints/affordances that the agent considers when planning those actions. At the third level, the operator is provided with information regarding the agent's projection of the future state, such as predicted consequences, likelihood of success/failure, and any uncertainty associated with the aforementioned projections. Incorporating all three levels should allow an operator to gain understanding of an agent's reasoning process behind its actions and help the operator make informed decisions as to whether he or she should intervene.

Recent research programs such as the U.S. Department of Defense Autonomy Research Pilot Initiative (ARPI) have started to investigate some of the key humanagent teaming issues that have to be addressed in order for mixed-initiative teams to perform effectively in the real world with all its complexities and unanticipated dynamics. This paper summarizes two efforts supported by the ARPI to investigate the effects of agent transparency on human-agent team performance in two military contexts. The first study, Autonomous Squad Member, deals with human interaction with a robotic partner in a simulated dismounted environment. The second study, IMPACT, describes human interaction with an intelligent agent to manage a team of heterogeneous robots. Preliminary results from each study are summarized to illustrate the utility of agent transparency for effectiveness of human-agent team performance.

\section{Autonomous Squad Member}

The objective of the Autonomous Squad Member (ASM) project is to investigate enabling agent capabilities to support military squad-level performance in dynamic mission environments [2]. The SAT model provided a framework for how to organize and implement the information coming from the ASM to the human squad members (Fig. 2). Ecological Interface Design (EID) and Cognitive Systems Engineering (CSE) principles were used to develop the visualizations of the SAT model-based information [1]. One of the principles of EID and CSE is to design an interface using metaphorical symbology that is based on principles that the user would already be familiar with [1]. The benefit of using a metaphorical representation, as an icon, is that it allows the user to use pre-existing knowledge while using the interface.

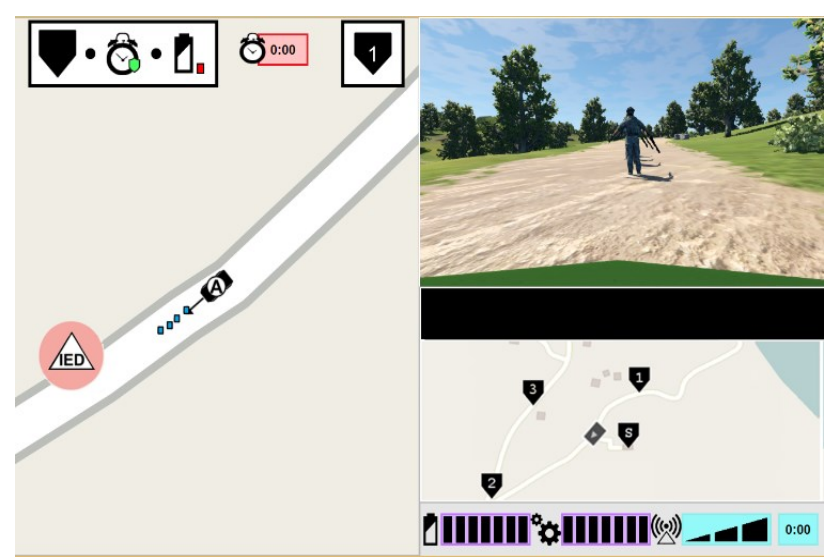

Figure 2: ASM experimental user interface

After the initial user interface was developed, in order to ensure the effectiveness of the designs, we conducted two usability evaluations: the pluralistic 
usability walkthrough [10] and the card sorting task [11]. The purpose of the pluralistic usability walkthrough is to have users and usability experts observe a typical use case of the interface and garner feedback on the tasks performed using the interface [10]. This feedback can be commentary on the design of the interface, areas of the task that the user might be having difficulty performing, or if the presentation of information in the training materials was unclear. The card sorting task was used to examine the mental models evoked by the symbology utilized in the interface [11].

Based on the results from these evaluations, a final user interface was developed, which will be used in a human-in-the-loop simulation experiment to further test the effects of ASM transparency on operator performance, workload, and trust in the ASM.

\section{IMPACT}

In the IMPACT study, we examined the level of information necessary to create an effective and transparent user interface to support human teaming with an intelligent agent (IA) for multi-robot

management in a series of simulated missions (Fig. 3). For each mission, the IA provided two recommended plans utilizing the robots (including ground, aerial, and surface platforms). Plan A was always the agent's top recommendation, and plan $B$ was the back-up plan. About $1 / 3$ of the time, Plan B was actually the better option-the agent was incorrect due to external information (changes in Commander's Intent, intelligence, etc.). A within-subjects design with three levels of agent transparency (based on the SAT model) was employed: Level $1+2$, Level $1+2+3$; Level $1+2+3+U$ (uncertainty information). Twenty-five young adults in central Florida (17 men; 8 women) between the ages of 18 and $36(M=23)$ participated in this study. Participants' task was to choose between Plan A and Plan $B$ based on the information presented by the IA and additional information that was provided (e.g., Commander's Intent, etc.).

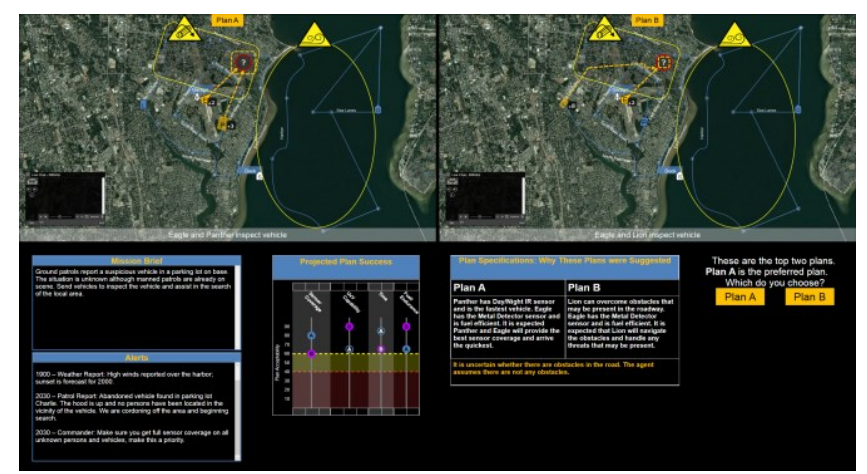

Figure 3: IMPACT experimental user interface.

Consistent with what we found in an earlier study using the same experimental paradigm, but different user interface designs [8], the results indicate that as agent transparency increased, so did operator performance (proper agent usage when the agent was correct and correct rejections when the agent was incorrect; Fig. 4). In other words, as the IA became more transparent, the participants were able to calibrate their trust in the IA's recommendations more effectively. Interestingly, the participants did not perceive themselves as significantly more situationally aware of their mission environments as the IA became more transparent (based on the subjective SART measures), although their task performance did improve. The increased operator performance did not appear to cause greater operator workload (based on NASA TLX), indicating the 
effectiveness of the user interface designs to convey the information to the operator without increasing operator workload. Indeed, our usability survey (System Usability Survey) indicates that as the IA's transparency level increased, so did the perceived usability of the IA-just as what we found in the prior study [8]. Regarding operator trust in the IA [6], there were no significant differences among the conditions. In other words, while participants' objective trust calibration (proper uses and correct rejections) improved as IA became more transparent, their subjective trust did not significantly increase similarly.

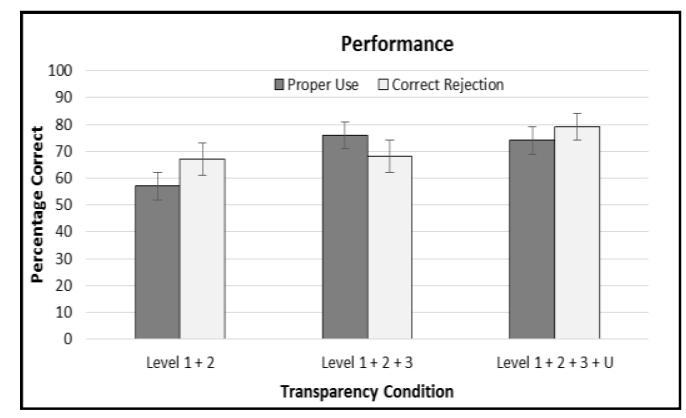

Figure 4: IMPACT - Operator performance.

\section{Acknowledgements}

This research is funded by the U.S. Department of Defense Autonomy Research Pilot Initiative (PM Dr. DH Kim). The authors wish to thank these individuals for their contribution: Daniel Barber, Jon Harris, Andrew Watson, Mike Rupp, Olivia Newton, Cintya Larios, MaryAnne Fields, Mark Draper, and Gloria Calhoun.

\section{References}

1. Kevin B. Bennett and John M. Flach. 2011. Display and Interface Design: Subtle Science, Exact Art. CRC Press, Boca Raton, FL.
2. Michael W. Boyce, Jessie Y. C. Chen, Anthony R. Selkowitz, Shan G. Lakhmani. 2015. Agent

Transparency for an Autonomous Squad Member (Tech Report: ARL-TR-7298). US Army Research Laboratory, Aberdeen Proving Ground MD.

3. Jessie Y. C. Chen and Michael J. Barnes. 2014. Human-agent teaming for multirobot control: A review of human factors issues. IEEE Trans. Human-Machine Systems 44, 1: 13-29.

4. Jessie Y. C. Chen, Katelyn Procci, Michael Boyce, Julia Wright, Andre Garcia, Michael Barnes. 2014 Situation awareness-based Agent Transparency (Tech Report: ARL-TR-6905). US Army Research Laboratory, Aberdeen Proving Ground MD.

5. Mica R. Endsley. 1995. Toward a theory of situation awareness in dynamic systems. Human Factors 37 1: 32-64.

6. Jiun-Yin Jian, Ann Bisantz, Colin Drury. 2000. Foundations for an empirically determined scale of trust in automated systems. Int J. Cognitive Ergonomics 4, 1: 53-71.

7. John D. Lee and Katrina A. See. 2004. Trust in technology: Designing for appropriate reliance. Human Factors 46, 1: 50-80.

8. Joseph Mercado, Michael Rupp, Jessie Chen, Daniel Barber, Katelyn Procci, Michael Barnes. In Press. Intelligent agent transparency in human-agent teaming for multi-UxV management. Human Factors.

9. Anand S. Rao and Michael P. Georgeff. 1995. BDI agents: From theory to practice. In Proc. Int. Conf. Multiagent Syst., 312-319.

10. Sirpa Riihiaho. 2002. The pluralistic usability walkthrough method. Ergo. in Design 10, 3: 23-27.

11. Donna Spencer. 2009. Card Sorting: Designing Usable Categories. Rosenfeld Media, NY, NY. 